\title{
Lateral Ventricular Meningioma Presenting with Intraventricular Hemorrhage
}

\author{
Ji Soo Moon, Seung Heon Cha, Won Ho Cho \\ Department of Neurosurgery, Pusan National University Hospital, Pusan National University School of Medicine, Busan, Korea
}

\author{
Received July 30, 2019 \\ Revised August 1, 2019 \\ Accepted September 27, 2019 \\ Correspondence \\ Seung Heon Cha \\ Department of Neurosurgery, \\ Pusan National University Hospital, \\ Pusan National University \\ School of Medicine, \\ 179 Gudeok-ro, Seo-gu, \\ Busan 49241, Korea \\ Tel: +82-51-240-7257 \\ Fax: +82-51-244-0282 \\ E-mail: neurocha@hanmail.net
}

\begin{abstract}
A 35-year-old female visited emergency department for a sudden onset of headache with vomiting after management for abortion at local department. Neurological examination revealed drowsy mentality without focal neurological deficits. CT showed $3.2 \times 3.4 \mathrm{~cm}$ hyperdense intraventricular mass with intraventricular hemorrhage. The intraventricular hemorrhage was found in lateral, 3rd, and 4th ventricles. MRI showed well enhancing intraventricular mass abutting choroid plexus in the trigone of the right lateral ventricle. CT angiography showed tortuous prominent arteries from choroidal artery in tumor. Her neurological status deteriorated to stupor and contralateral hemiparesis during planned preoperative workup. Urgent transtemporal and transcortical approach with decompressive craniectomy for removal of intraventricular meningioma with hemorrhage was done. Grossly total removal of ventricular mass was achieved. Pathological finding was meningotheliomatous meningioma of World Health Organization (WHO) grade I. The patient recovered to alert mentality and no motor deficit after intensive care for increased intracranial pressure. However, visual field defect was developed due to posterior cerebral artery territory infarction. The visual deficit did not resolve during follow up period. Lateral ventricular meningioma with spontaneous intraventricular hemorrhage in pregnant woman is very uncommon. We report a surgical case of lateral ventricular meningioma with rapid neurological deterioration for intraventricular hemorrhage.
\end{abstract}

Key Words Meningioma; Hemorrhage; Pregnancy.

\section{INTRODUCTION}

Lateral ventricular meningiomas are rare incidence of approximately $0.5-3 \%$ out of all intracranial meningioma $[1,2]$. The ventricular meningioma is often one of the incidentally discovered tumor on brain MRI. They usually have no definite clinical symptoms when they are small. However, they often present with chronic increased intracranial pressure, visual field defects, ataxia, and memory impairment as they grow larger [3,4]. Intracranial hemorrhage from brain tumor has been well known in patients with malignant brain tumors such as malignant glioma, metastatic carcinoma, and choroid plexus papilloma. Benign brain tumors with intracranial hemorrhage are rare. Lateral ventricular meningioma

This is an Open Access article distributed under the terms of the Creative Commons Attribution Non-Commercial License (https://creativecommons.org/licenses/by-nc/4.0) which permits unrestricted non-commercial use, distribution, and reproduction in any medium, provided the original work is properly cited.

Copyright $\odot 2019$ The Korean Brain Tumor Society, The Korean Society for NeuroOncology, and The Korean Society for Pediatric Neuro-Oncology presenting with acute intraventricular hemorrhage is considered more uncommon. The lateral ventricular meningioma with acute hemorrhage is exceedingly rare in woman with regard to pregnancy. To identify this type of meningioma, we review and evaluate the clinical and surgical features of the meningioma and the relevant literatures.

\section{CASE REPORT}

A 35-year-old female visited emergency department for a sudden onset of headache and vomiting followed by altered sensorium after management for abortion at local department of obstetrics. Neurological examination on admission revealed drowsy mentality without focal neurological deficits. Blood pressure on admission was 120/80 $\mathrm{mm} \mathrm{Hg}$. The pressure was stable until aggravation of neurological status. On admission, biochemical markers were within normal limit. The coagulation profile from hematological analysis was normal. CT showed $3.2 \times 3.4 \mathrm{~cm}$ hyperdense intraventricular mass with sur- 
rounding intraventricular hemorrhage. The intraventricular hemorrhage was found in right lateral, $3 \mathrm{rd}$, and 4th ventricles (Fig. 1). MRI showed well enhanced intraventricular mass abutting choroid plexus in the trigone of the right lateral ventricle (Fig. 2). CT angiography showed tortuous prominent arteries from choroidal arteries in the tumor (Fig. 3). She was recovered to alert mentality with intermittent headache by medical treatment for increased intracranial pressure. However, rapid neurological deterioration with signs of transtentorial herniation in spite of medical management was developed during planned preoperative workup for elective surgery. Urgent transtemporal transcortical approach with decompressive craniectomy was done. Hypervascular tumor with capsule was exposed through enlarged ipsilateral temporal horn after temporal lobectomy. The tumor adhered slightly to the walls of the lateral ventricle. Feeding arteries from choroidal arteries were coagulated initially. The tumor was completely removed after central debulking and marginal dissection (Fig. 4). We removed and irrigated large amount of clot in cerebrospinal fluid of the ventricle after total removal of the mass. The mass was pathologically diagnosed as meningotheliomatous meningioma (World Health Organization grade I) (Fig. 5). She recovered well after sedative treatment at intensive care unit to control refractory intracranial hypertension. Left homonymous hemianopsia was developed after surgery and did not resolve during follow up period. The patient provided written informed consent for this study.

\section{DISCUSSION}

The intraventricular meningioma is $0.5-5 \%$ of all intracranial meningiomas with $80 \%$ presenting in the lateral ventricles, $15 \%$ in the third ventricle, and $5 \%$ in the fourth ventricle [5]. Over $90 \%$ of meningioma in the lateral ventricle occurs in the trigone $[3,4]$. Lateral ventricular meningiomas are derived from the arachnoid of the choroidal plexus or the outer membrane of vessels in the lateral ventricles. The feeding artery can be the anterior or posterior choroidal artery, and the lenticulostriate artery or deep perforating arteries from the thalamus may also contribute, especially with large tumors. At present, preoperative CT angiography and MR angiography are enough to evaluate blood supply and vascular structure of the tumor. Preoperative CT angiography of our case showed tortuous feeding arteries at inferomedial compartment of the tumor.

The clinical manifestations of lateral ventricular meningiomas depend mainly on tumor size. A small tumor usually has no symptoms because of a relatively large compensating space
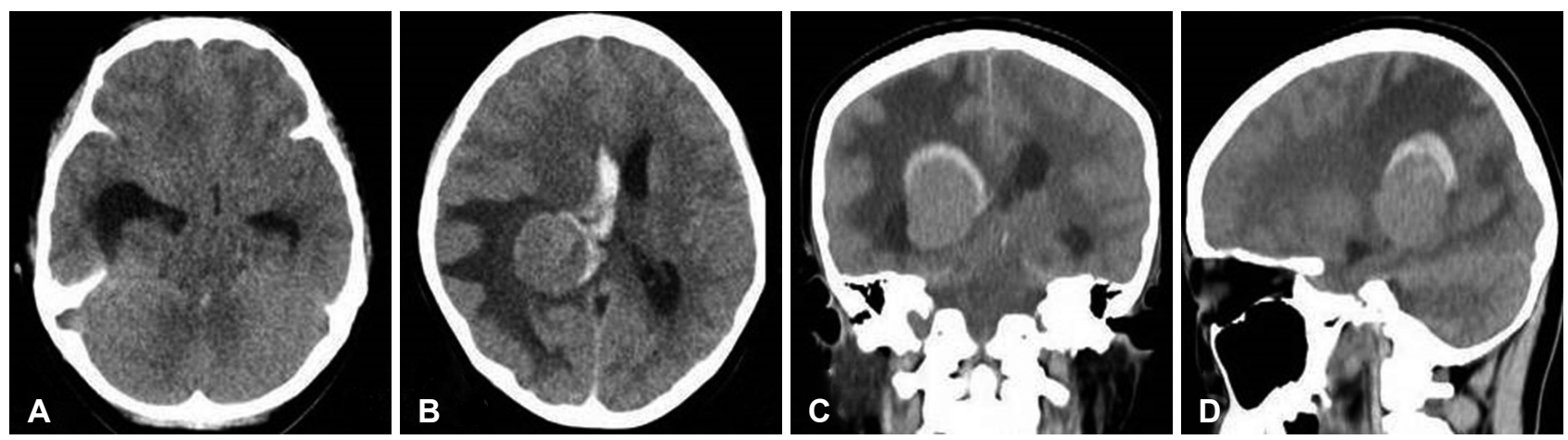

Fig. 1. Preoperative axial $(A, B)$, coronal $(C)$, and sagittal $(D) C T$ showing a homogenous mass in trigone of right lateral ventricle surrounded by hemorrhage with enlargement of ipsilateral temporal horn.
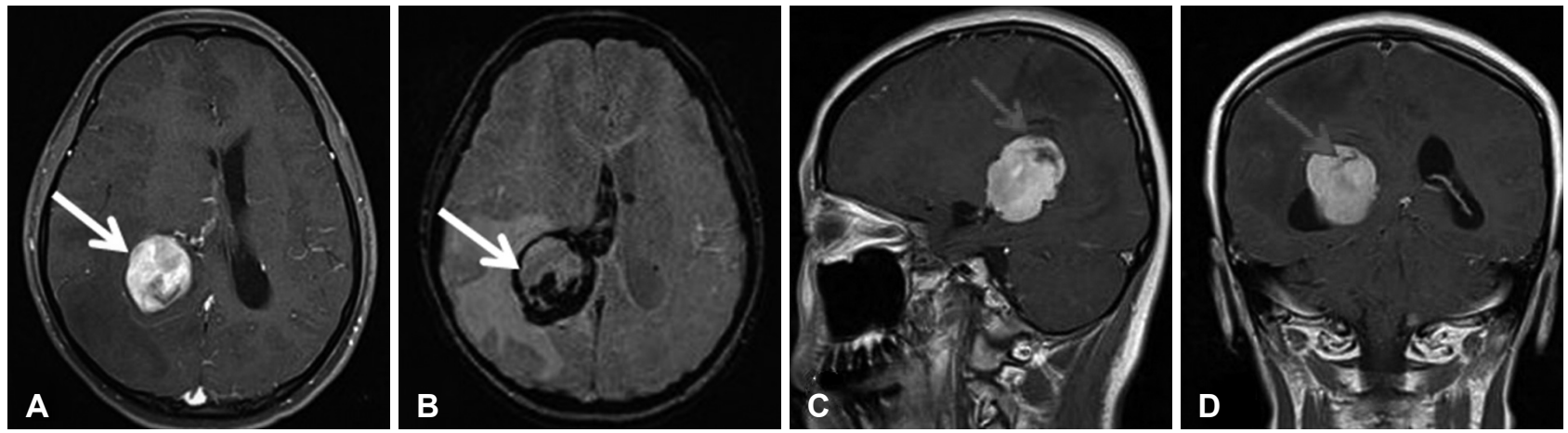

Fig. 2. Preoperative axial (A), susceptibility weighted imaging (B), sagittal (C), and coronal (D) enhanced MRI showing homogenous enhancing mass in right trigone with hemorrhage (arrow) from the tumor periphery. 

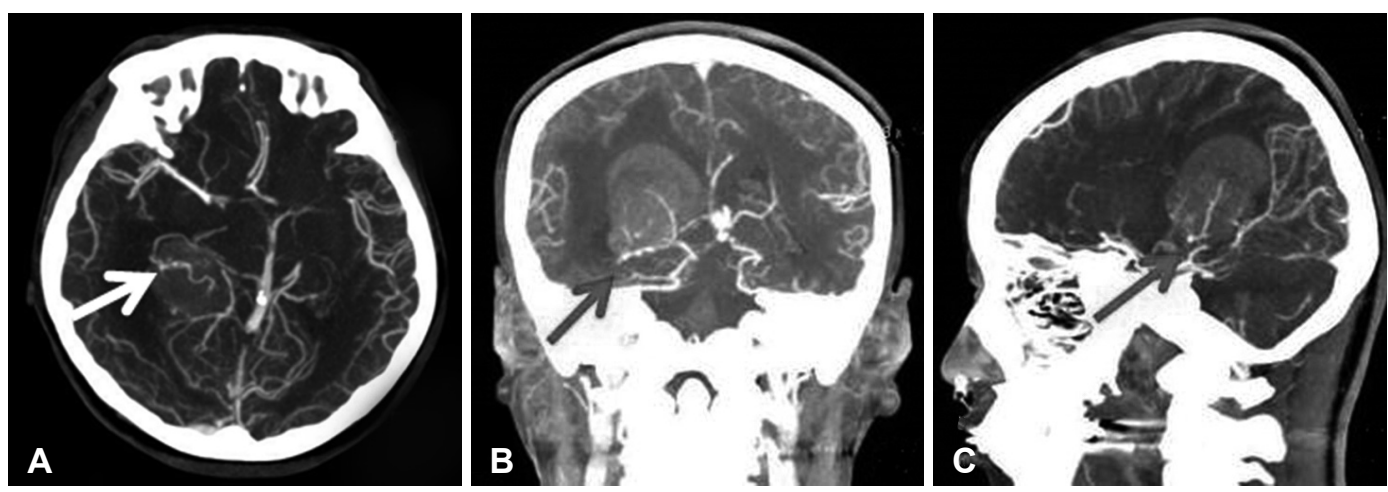

Fig. 3. Axial (A), coronal (B), and sagittal (C) enhanced CT angiography showing enlarged tortuous feeding artery (arrow) from anterior choroidal artery.
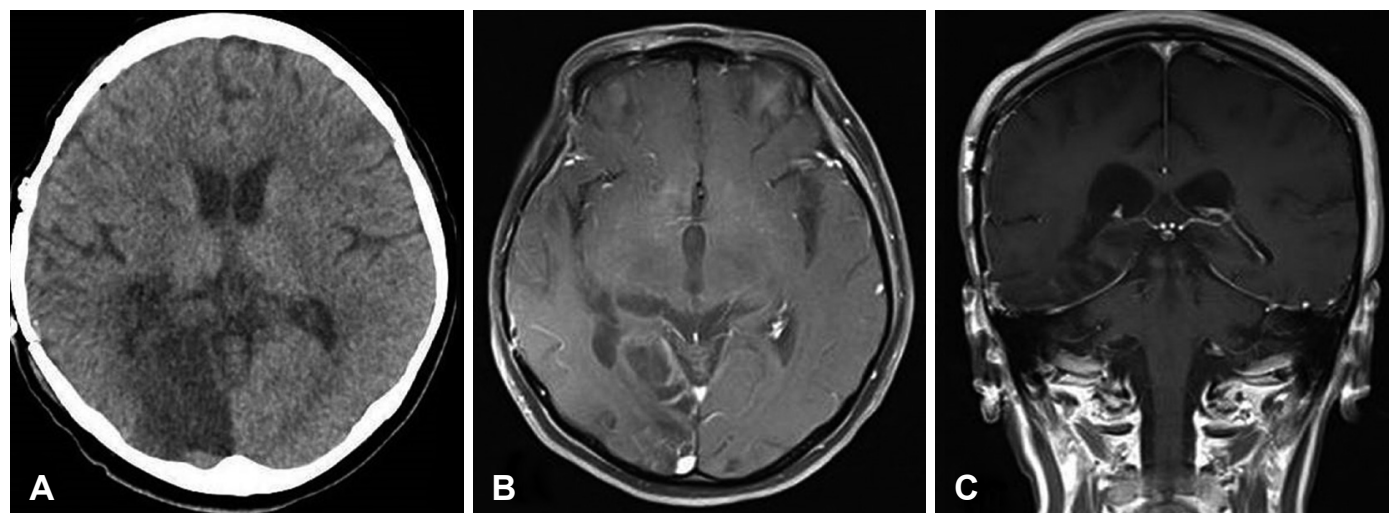

Fig. 4. Postoperative $C T(A)$ and $M R I(B, C)$ showing total removal of trigonal meningioma with infarction of right occipital lobe for occlusion of posterior cerebral artery from preoperative transient transtentorial herniation.
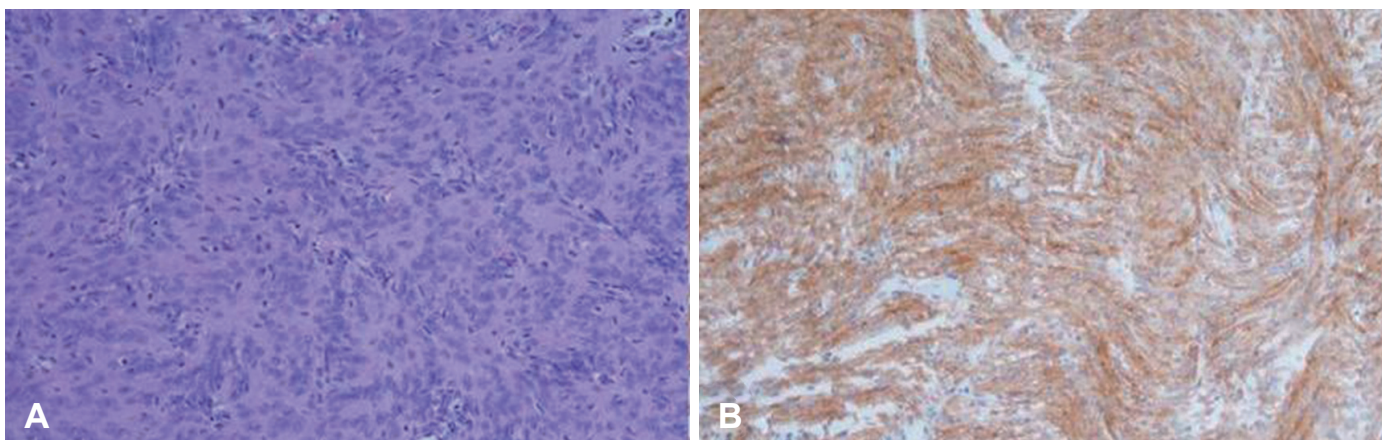

Fig. 5. Histopathological findings. World Health Organization grade I meningotheliomatous meningioma with meningothelial cells exhibiting whorled growth (hematoxylin and eosin staining, $\times 200)(A)$ and epithelial membrane antigen $(+)(\times 200)(B)$.

of the lateral ventricle. We frequently found small ventricular tumors as incidentally asymptomatic tumor. Meningiomas are most often slow-growing tumors and usually present with symptoms such as headache, dizziness, seizures, and gradually progressing neurologic deficits when the tumor is large. Bleeding in a malignant brain tumor generally results from weakness of the neoplastic vessels, infiltration of tumor cells into the vessel, and tendency of the mural endothelium to proliferate, which lead to vessel destruction and necrosis [6-8]. However, The clinical presentation of spontaneous hemorrhage in benign tumors is rare. Lateral ventricular meningiomas present- ing with intraventricular hemorrhage are extremely rare. The mechanisms associated with spontaneous hemorrhage from nonmalignant meningiomas apparently are unknown [9]. Although rare, intraventicular hemorrhage of our case produced a life-threatening event owing to acute increased intracranial pressure and transtentorial herniation. Several hypotheses about hemorrhage from intraventricular meningioma has been proposed. First, the vascular network within the meningioma may rupture due to its abnormal development. Second, a rapidly growing meningioma or internal necrosis after venous thrombosis may lead to hemorrhage. Third, arteries that 
feed the meningioma are dilated and tortuous and thus lose the ability to regulate blood pressure fluctuation. Fourth, expansive growth of the meningioma stretches and tears bridging veins [10-13]. Other factors, such as coagulation disorder inside the meningioma, peritumoral edema, infarction within the tumor, and malignant transformation of the tumor may also result in hemorrhage of intracranial meningiomas. Of these hypotheses, the first one is the most widely accepted, but it still cannot explain all meningioma hemorrhages because not all meningiomas demonstrate abnormal blood vessel development. Thus, a combination of the aforementioned factors may play a part in this process [14].

The present case revealed the followings: benign meningotheliomatous meningioma was pathologically diagnosed; dilated tortuous vessels in the tumor was shown by preoperative CT angiography; and neurological symptoms were developed after obstetric procedure with possibility of blood pressure fluctuation. Therefore, we concluded that the hemorrhage in this intraventricular meningioma resulted from rupture of blood vessels with dilated tortuous development, and that the hemorrhage infiltrated the tumor tissues due to the pressure gradient. Accordingly, the first and third hypotheses best explain the hemorrhage of this meningioma.

Our case developed intraventricular hemorrhage from meningioma after obstetric procedure for abortion. Lusis et al. [15] reported the clinicopathologic study about pregnancy associated meningioma. They suggested potentially reversible hemodynamic changes such as intratumoral hypervascularity, edema, and spontaneous hemorrhage or necrosis in pregnancy associated meningioma [15]. There was some evidence to suggest that progesterone increases cerebrovascular inflammation and vascular dilatation [16]. We did not consider the presence of residual or unresected meningioma to be a contraindication to pregnancy. However, based on our case and the available literature, for those where further increases in tumor size or associated edema could be associated with significant morbidity or even mortality, surgical or radiosurgical therapy should be carefully discussed before getting pregnant to prevent significant morbidity or mortality of women of childbearing age with meningioma.

Trigone is deep anatomical location surrounded by important eloquent structures, mainly including the optic radiation in the temporal lobe, the motor and sensory centers in the parietal lobe, and the sensory speech center in the dominant hemisphere. Due to the fluid of ventricular cavity and the slow growth rate, most tumors are quite large and hypervascularized before development of any symptoms [5]. The optimal surgical approach for trigonal meningioma is still controversial. The present approaches include the transtemporal approach through the middle or inferior temporal gyrus, the transparietal approach through the superior parietal lobe, and the interhemispheric parietooccipital precuneus (parasplenial) approach. All the approaches have individual advantages and disadvantages. Of all the approaches, the transcortical approach trough parietooccipital sulcus is the most preferred route for large lateral ventricular meningioma despite of cortical damage. Brain CT of our case after neurological deterioration with signs of impending transtentorial herniation showed peritumoral edema and enlarged ipsilateral temporal horn caused by tumor in trigone. We took emergent transtemporal approach with decompressive craniectomy to overcome mortality and morbidiy of transtentorial herniation. Urgent imaging study was quite helpful for preoperative evaluation to make a best surgical plan.

Lateral ventricular meningioma is often asymptomatic because the lateral ventricle has a relatively large compensating space. However, hemodynamic alterations associated with pregnancy could produce hemorrhage in clinically silent meningioma. When the ventricular meningioma rarely produces hemorrhage and remarkable symptom such as increased intracranial pressure, particular attention and rapid surgical intervention are warranted to avoid complications for delayed treatment.

\section{Conflicts of Interest}

The authors have no potential conflicts of interest.

\section{Acknowledgments}

This work was supported by 2-year Research Grant of Pusan National University.

\section{REFERENCES}

1. Nakamura M, Roser F, Bundschuh O, Vorkapic P, Samii M. Intraventricular meningiomas: a review of 16 cases with reference to the literature. Surg Neurol 2003;59:491-503; discussion 503-4.

2. Kim EY, Kim ST, Kim HJ, Jeon P, Kim KH, Byun HS. Intraventricular meningiomas: radiological findings and clinical features in 12 patients. Clin Imaging 2009;33:175-80.

3. Lyngdoh BT, Giri PJ, Behari S, Banerji D, Chhabra DK, Jain VK. Intraventricular meningiomas: a surgical challenge. J Clin Neurosci 2007;14:442-8.

4. Bertalanffy A, Roessler K, Koperek O, et al. Intraventricular meningiomas: a report of 16 cases. Neurosurg Rev 2006;29:30-5.

5. Ma J, Cheng L, Wang G, Lin S. Surgical management of meningioma of the trigone area of the lateral ventricle. World Neurosurg 2014;82:757-69.

6. Bosnjak R, Derham C, Popović M, Ravnik J. Spontaneous intracranial meningioma bleeding: clinicopathological features and outcome. J Neurosurg 2005;103:473-84.

7. Niiro M, Ishimaru K, Hirano H, Yunoue S, Kuratsu J. Clinico-pathological study of meningiomas with haemorrhagic onset. Acta Neurochir (Wien) 2003;145:767-72.

8. Vij M, Jaiswal S, Jaiswal AK, Kumar S, Behari S. Meningioma with hemorrhagic onset: two case reports. J Cancer Res Ther 2012;8:145-7.

9. Kim JH, Gwak HS, Hong EK, Bang CW, Lee SH, Yoo H. A case of benign meningioma presented with subdural hemorrhage. Brain Tumor Res Treat 2015;3:30-3.

10. Helle TL, Conley FK. Haemorrhage associated with meningioma: a 
case report and review of the literature. J Neurol Neurosurg Psychiatry 1980;43:725-9.

11. Gruszkiewicz J, Doron Y, Gellei B, Peyser E. Massive intracerebral bleeding due to supratentorial meningioma. Neurochirurgia (Stuttg) 1969;12:107-11.

12. Bloomgarden GM, Byrne TN, Spencer DD, Heafner MD. Meningioma associated with aneurysm and subarachnoid hemorrhage: case report and review of the literature. Neurosurgery 1987;20:24-6.

13. Martínez-Lage JF, Poza M, Martínez M, Esteban JA, Antúnez MC,
Sola J. Meningiomas with haemorrhagic onset. Acta Neurochir (Wien) 1991;110:129-32.

14. Kim DG, Park CK, Paek SH, et al. Meningioma manifesting intracerebral haemorrhage: a possible mechanism of haemorrhage. Acta Neurochir (Wien) 2000;142:165-8.

15. Lusis EA, Scheithauer BW, Yachnis AT, et al. Meningiomas in pregnancy: a clinicopathologic study of 17 cases. Neurosurgery 2012;71:951-61.

16. Krause DN, Duckles SP, Pelligrino DA. Influence of sex steroid hormones on cerebrovascular function. J Appl Physiol (1985) 2006;101:1252-61. 